PROCEEDINGS OF THE

AMERICAN MATHEMATICAL SOCIETY

Volume 136, Number 9, September 2008, Pages 3121-3131

S 0002-9939(08)09059-X

Article electronically published on April 22, 2008

\title{
ON A BOUNDARY ANALOGUE OF THE CARATHÉODORY-SCHUR INTERPOLATION PROBLEM
}

\author{
VLADIMIR BOLOTNIKOV
}

(Communicated by Joseph A. Ball)

\begin{abstract}
Characterization of Schur functions in terms of their Taylor coefficients is due to C. Carathéodory and I. Schur. We discuss the boundary analogue of this problem.
\end{abstract}

\section{INTRODUCTION}

Characterization of Schur functions (analytic self-maps of the unit disk $\mathbb{D}$ ) in terms of their Taylor coefficients goes back to I. Schur 14] (and to C. Carathéodory for a related class of functions). It looks natural to consider a similar question in the "boundary" setting:

Problem 1.1. Given a point $t_{0}$ on the unit circle $\mathbb{T}$ and given complex numbers $c_{0}, \ldots, c_{n}$, find necessary and sufficient conditions for existence of a Schur function $f$ that admits the asymptotic expansion

$$
f(z)=c_{0}+c_{1}\left(z-t_{0}\right)+\ldots+c_{n}\left(z-t_{0}\right)^{n}+o\left(\left(z-t_{0}\right)^{n}\right)
$$

as $z$ tends to $t_{0}$ nontangentially.

The class of all Schur functions will be denoted by $\mathcal{S}$. Note that condition (1.1) is equivalent to existence of nontangential (angular) boundary limits for $f^{(j)}(z)$ at $t_{0}$ and equalities

$$
\lim _{z \rightarrow t_{0}} \frac{f^{(j)}(z)}{j !}=c_{j} \quad \text { for } j=1, \ldots, n .
$$

Since $c_{0}$ is equal to the angular boundary limit of a Schur function, condition $\left|c_{0}\right| \leq 1$ is necessary for (1.2) to hold for some $f \in \mathcal{S}$. If $n=0$, this condition is also sufficient and there are infinitely many Schur functions $f$ with the angular limit at $t_{0}$ equals $c_{0}$. For $n=1$, the answer is less trivial but still simple:

Given $t_{0} \in \mathbb{T}$ and $c_{0}, c_{1} \in \mathbb{C}$, there exists an $f \in \mathcal{S}$ such that

$$
\lim _{z \rightarrow t_{0}} f(z)=c_{0} \quad \text { and } \quad \lim _{z \rightarrow t_{0}} f^{\prime}(z)=c_{1}
$$

if and only if either $\left|c_{0}\right|<1$ or $\left|c_{0}\right|=1$ and $t_{0} c_{1} \bar{c}_{0} \geq 0$.

Moreover, if the necessary conditions are satisfied, then the problem (1.3) has infinitely many solutions, unless $\left|c_{0}\right|=1$ and $c_{1}=0$ in which case the problem

Received by the editors December 27, 2005, and, in revised form, December 29, 2006.

2000 Mathematics Subject Classification. Primary 47A57.

Key words and phrases. Schur functions, boundary interpolation problem.

(C)2008 American Mathematical Society Reverts to public domain 28 years from publication 
has the unique solution $f \equiv c_{0}$. Indeed, if $\left|c_{0}\right|<1$, then there are infinitely many functions $f \in \mathcal{S}$ subject to (1.3), by Theorem 1.2 below. If $\left|c_{0}\right|=1$, then the problem (1.3) has a solution if and only if the number $d:=t_{0} c_{1} \bar{c}_{0}$ is nonnegative, by the Carathéodory-Julia theorem [6]. If $d=0$ (or equivalently, if $c_{1}=0$ ), the uniqueness follows by Julia's lemma [7. If $d>0$, there is a linear fractional parametrization with free Schur class parameter of all functions $f \in \mathcal{S}$ satisfying (1.3) (see 12]).

If $n \geq 2$, the answer for Problem 1.1 is not known. The next theorem might be the first step toward it (for a simple proof in the context of matrix valued Schur functions, see [10, Proposition 5.2]).

Theorem 1.2. Let $t_{0} \in \mathbb{T}, c_{0}, \ldots, c_{n} \in \mathbb{C}$ and let $\left|c_{0}\right|<1$. Then there exist infinitely many Schur functions $f$ satisfying interpolation conditions (1.2).

Somewhat greater effort is involved to prove a similar result for the multi-point boundary interpolation problem for operator valued Schur functions. Such problems have arisen in the contexts of robust control system synthesis [11] and of partial realization problem [10. In what follows, $\mathcal{S}(\mathcal{X}, \mathcal{Y})$ denotes the Schur class of $\mathcal{L}(\mathcal{X}, \mathcal{Y})$-valued functions $f$ analytic on $\mathbb{D}$ with $\|f\|_{\infty}:=\sup _{z \in \mathbb{D}}\|f(z)\|_{o p} \leq 1$. The symbol $\mathcal{L}(\mathcal{X}, \mathcal{Y})$ stands for the algebra of bounded linear operators mapping a Hilbert space $\mathcal{X}$ into another Hilbert space $\mathcal{Y}$ and we shall shorten $\mathcal{L}(\mathcal{Y}, \mathcal{Y})$ to $\mathcal{L}(\mathcal{Y})$. The symbol $I$ will stand for the identity operator acting on an appropriate Hilbert space.

Problem 1.3. Given $k$ distinct points $t_{1}, \ldots, t_{k} \in \mathbb{T}$, nonnegative integers $n_{1}, \ldots, n_{k}$ and $N=\sum_{i=1}^{k}\left(n_{i}+1\right)$ operators $c_{i j} \in \mathcal{L}(\mathcal{X}, \mathcal{Y})$, find necessary and sufficient conditions for existence of a Schur function $f \in \mathcal{S}(\mathcal{X}, \mathcal{Y})$ such that

$$
\lim _{z \rightarrow t_{i}} \frac{f^{(j)}(z)}{j !}=c_{i j} \quad \text { for } i=1, \ldots, k ; j=0, \ldots, n_{i},
$$

where the angular limits are understood in the weak sense.

Conditions $\left\|c_{i 0}\right\| \leq 1(i=1, \ldots, k)$ are necessary for existence of an $f \in \mathcal{S}(\mathcal{X}, \mathcal{Y})$ satisfying (1.4). Sufficient (not necessary) conditions are given in the next theorem that generalizes Theorem 1.2 and is the main result of this note. Note that the case when $n_{i}=0$ for $i=1, \ldots, k$ (i.e., the boundary Nevanlinna-Pick problem) was considered in [11] (see Theorem 1.5 there) and discussed in [2, Chapter 21].

Theorem 1.4. Given the data as above, let us assume that

$$
\left\|c_{i 0}\right\|<1 \quad \text { for } i=1, \ldots, k \text {. }
$$

Then there exist infinitely many Schur functions $f \in \mathcal{S}(\mathcal{X}, \mathcal{Y})$ satisfying interpolation conditions (1.4).

Moreover, we will show that there exist infinitely many rational functions $f \in$ $\mathcal{S}(\mathcal{X}, \mathcal{Y})$ subject to (1.4) that are analytic and with $\|f\|_{\infty} \leq 1$ on a larger disk $\mathbb{D}_{\rho}$ $(\rho>1)$; for such functions all the limits in (1.4) are uniform and can be replaced by the values of $f^{(j)}$ at $t_{i}$.

In conclusion we recall some other boundary interpolation problems related to Problem 1.3 which were considered previously and which provide some other sufficient conditions (different from (1.5) ) for Problem 1.3 to have a solution. These 
conditions actually guarantee the existence of a rational inner (bi-inner, in the operator valued setting) solution of the problem and therefore they are too far from being necessary. For simplicity, we will discuss only the scalar valued setting and we will assume that all the integers $n_{i}$ 's be odd: $n_{i}=2 m_{i}+1$, for $i=1, \ldots, k$. If there exists a finite Blaschke product $f$ satisfying conditions (1.4), then it is readily seen that $\left|c_{i, 0}\right|=1$ for $i=1, \ldots, k$ and that the matrix

$$
\mathbb{P}=\left[\left[\left.\frac{1}{\ell ! r !} \frac{\partial^{\ell+r}}{\partial z^{\ell} \partial \bar{\zeta}^{r}}\left(\frac{1-f(z) \overline{f(\zeta)}}{1-z \bar{\zeta}}\right)\right|_{\begin{array}{l}
z=t_{i}, \\
\zeta=t_{j}
\end{array}}\right]_{\begin{array}{l}
\ell=0, \ldots, m_{i} \\
r=0, \ldots, m_{j}
\end{array}}\right]_{i, j=1}^{k}
$$

is well defined and can be expressed explicitly in terms of the data $\left\{t_{j}, c_{i j}\right\}$ (we refer to [5, Section 3] for the explicit formulas). Since $f \in \mathcal{S}$, the latter matrix is positive semidefinite. Interpolation problems studied in [1- [5], 9, , 12, 13] can be formulated as follows:

Given the data $\left\{t_{i}, m_{i}, c_{i j}\right\}$ such that

$$
\mathbb{P} \geq 0 \quad \text { and } \quad\left|c_{i, 0}\right|=1 \quad(i=1, \ldots, k),
$$

(1) Find all Schur functions $f$ satisfying conditions (1.4) or

(2) Find all Schur functions $f$ satisfying conditions (1.4) for $i=1, \ldots, k$; $j=0, \ldots, 2 m_{i}$ (recall that $\left.2 m_{i}=n_{i}-1\right)$ and

$$
(-1)^{m_{i}} t_{i}^{2 m_{i}+1}\left(c_{i, 2 m_{i}+1}-\lim _{z \rightarrow t_{i}} \frac{f^{\left(2 m_{i}+1\right)}(z)}{\left(2 m_{i}+1\right) !}\right) \bar{c}_{i, 0} \geq 0 \quad \text { for } i=1, \ldots, k .
$$

The second problem is a relaxed version of the first one when the equalities assigning the values for the boundary limits of $f^{\left(n_{i}\right)}$ at $t_{i}$ in (1.4) are replaced by inequalities (1.7). The case when $\mathbb{P}$ is singular is not very interesting in the scalar valued setting: the relaxed Problem 2 has a unique solution (necessarily a finite Blaschke product) which may be or may be not a solution of Problem 1. If $\mathbb{P}>0$, then both problems have infinitely many solutions that can be parametrized in terms of a linear fractional transformation with the free Schur class parameter (Problem 2; see [1]- 5], 12]) or with Schur class parameters satisfying certain constraints (Problem 1; see [13, [9], 5]). In particular, it follows that conditions (1.6) (with $\mathbb{P}$ invertible) are sufficient for Problem 1.3 to have a solution.

\section{Proof of Theorem 1.4}

To prove Theorem 1.4, we shall use approximation arguments from 2, Chapter 21] a brief discussion there shows how to get Theorem 1.4 for the case $n_{i}=0$ $(i=1, \ldots, k)$ from the classical Nevanlinna-Pick theorem. An auxiliary result (see Lemma 2.2 below) will enable us to apply similar arguments for the general case. The possibility to apply approximation arguments for a general multi-point interpolation problem was pointed out in [11, Remark 1.7]. First we recall a well known result on the multi-point Carathéodory-Schur problem when all the interpolation nodes fall inside the unit disk. The data set for this problem

$$
\Sigma=\left\{\mathbf{z}, \mathbf{n}, C_{\mathbf{n}}(\mathbf{z})\right\}
$$

consists of $k$-tuples $\mathbf{z}=\left\{z_{1}, \ldots, z_{k}\right\}$ of distinct points in $\mathbb{D}$ and $\mathbf{n}=\left\{n_{1}, \ldots, n_{k}\right\}$ of nonnegative integers and of operators $s_{0}\left(z_{i}\right), \ldots, s_{n_{i}}\left(z_{i}\right) \in \mathcal{L}(\mathcal{X}, \mathcal{Y})$ assigned to 
each pair $\left(z_{i}, n_{i}\right)$ that can be arranged in the matrix form as

$$
C_{\mathbf{n}}(\mathbf{z})=\left[\begin{array}{c}
C_{n_{1}}\left(z_{1}\right) \\
\vdots \\
C_{n_{k}}\left(z_{k}\right)
\end{array}\right], \quad \text { where } \quad C_{n_{i}}\left(z_{i}\right)=\left[\begin{array}{c}
s_{0}\left(z_{i}\right) \\
\vdots \\
s_{n_{i}}\left(z_{i}\right)
\end{array}\right] .
$$

Associated with the data (2.1) is the Carathéodory-Pick operator $P_{\Sigma}$ defined as a unique solution of the Stein equation

$$
P_{\Sigma}-\mathcal{J}_{\mathbf{n}}(\mathbf{z}) P_{\Sigma} \mathcal{J}_{\mathbf{n}}(\mathbf{z})^{*}=E_{\mathbf{n}} E_{\mathbf{n}}^{*}-C_{\mathbf{n}}(\mathbf{z}) C_{\mathbf{n}}(\mathbf{z})^{*}
$$

where

$$
\mathcal{J}_{\mathbf{n}}(\mathbf{z})=\left[\begin{array}{ccc}
\mathcal{J}_{n_{1}}\left(z_{1}\right) & & 0 \\
& \ddots & \\
0 & & \mathcal{J}_{n_{k}}\left(z_{k}\right)
\end{array}\right] \quad \text { and } \quad E_{\mathbf{n}}=\left[\begin{array}{c}
E_{n_{1}} \\
\vdots \\
E_{n_{k}}
\end{array}\right]
$$

In (2.4) and in what follows, $\mathcal{J}_{n}(z): \mathcal{Y}^{n+1} \rightarrow \mathcal{Y}^{n+1}$ and $E_{n}: \mathcal{Y} \rightarrow \mathcal{Y}^{n+1}$ are the operators defined by

$$
\mathcal{J}_{n}(z)=\left[\begin{array}{cccc}
z I_{\mathcal{Y}} & 0 & \ldots & 0 \\
I_{\mathcal{Y}} & z I_{\mathcal{Y}} & \ddots & \vdots \\
& \ddots & \ddots & 0 \\
0 & & I_{\mathcal{Y}} & z I_{\mathcal{Y}}
\end{array}\right] \text { and } E_{n}=\left[\begin{array}{c}
I_{\mathcal{Y}} \\
0 \\
\vdots \\
0
\end{array}\right]
$$

for $z \in \mathbb{C}$ and $n \in \mathbb{Z}_{+}$. Equation (2.3) indeed has a unique solution since $\operatorname{spec} \mathcal{J}_{\mathbf{n}}(\mathbf{z})$ $=\left\{z_{1}, \ldots, z_{k}\right\} \subset \mathbb{D}$. Taking adjoints in (2.3) we see that $P_{\Sigma}^{*}$ also satisfies (2.3) and then by uniqueness, $P_{\Sigma}=P_{\Sigma}^{*}$. The following interpolation result is well known (see e.g., [2, Chapter 18], [8]).

Theorem 2.1. There exists a Schur function $h \in \mathcal{S}(\mathcal{X}, \mathcal{Y})$ such that

$$
\frac{h^{(j)}\left(z_{i}\right)}{j !}=s_{j}\left(z_{i}\right) \quad \text { for } i=1, \ldots, k ; j=0, \ldots, n_{i}
$$

if and only if the Carathéodory-Pick operator $P_{\Sigma}$ associated with the data (2.1) is positive semidefinite. Furthermore, if $P_{\Sigma}$ is strictly positive definite (s.p.d.), there exist infinitely many rational Schur functions satisfying conditions (2.6).

Now let us assume that the points $z_{1}, \ldots, z_{k} \in \mathbb{D}$ are of the form $z_{i}=r t_{i}$ for $i=1, \ldots, k$, where $t_{1}, \ldots, t_{k}$ are fixed points on $\mathbb{T}$ and $r \in(0,1)$ is a parameter. Thus, $\mathbf{z}=r \mathbf{t}=\left(r t_{1}, \ldots, r t_{k}\right)$. Since we are interested in $z_{i}$ 's that are close enough to $t_{i}$ 's, we may assume that $r \in(\rho, 1)$ for some $\rho>0$. Consequently, the operators $s_{j}\left(z_{i}\right)$ are replaced by functions $s_{j}\left(r t_{i}\right):=s_{i j}(r):(\rho, 1) \rightarrow \mathcal{L}(\mathcal{X}, \mathcal{Y})$ for $i=1, \ldots, k$ and $j=0, \ldots, n_{i}$ that still can be arranged in the matrix form as in (2.2):

$$
C_{\mathbf{n}}(r)=\left[\begin{array}{c}
C_{n_{1}}(r) \\
\vdots \\
C_{n_{k}}(r)
\end{array}\right], \quad \text { where } \quad C_{n_{i}}(r)=\left[\begin{array}{c}
s_{i 0}(r) \\
\vdots \\
s_{i, n_{i}}(r)
\end{array}\right] .
$$

Given such data

$$
\Sigma(r)=\left\{\mathbf{z}=r \mathbf{t} \mathbf{n}, C_{\mathbf{n}}(r)\right\} \quad(\rho<r<1)
$$


depending on the parameter $r$, one can define the Carathéodory-Pick operator $P_{\Sigma(r)}$ for every $r \in(\rho, 1)$ as the unique solution of the Stein equation

$$
P_{\Sigma(r)}-\mathcal{J}_{\mathbf{n}}(r \mathbf{t}) P_{\Sigma(r)} \mathcal{J}_{\mathbf{n}}(r \mathbf{t})^{*}=E_{\mathbf{n}} E_{\mathbf{n}}^{*}-C_{\mathbf{n}}(r) C_{\mathbf{n}}(r)^{*} .
$$

Lemma 2.2. Given the data $\Sigma(r)$ as in (2.8), let us assume that there exist positive numbers $\delta<1$ and $M$ such that

$$
\left\|s_{i, 0}(r)\right\| \leq \delta \quad \text { and } \quad\left\|s_{i, j}(r)\right\| \leq M
$$

for all $j=1, \ldots, n_{i}, i=1, \ldots, k$ and $r \in(\rho, 1)$. Then there exists $r_{0}\left(\rho<r_{0}<1\right)$ such that the Carathéodory-Pick operator $P_{\Sigma(r)}$ is s.p.d. for every $r \in\left(r_{0}, 1\right)$.

The proof of this lemma will be given in the next section.

Proof of Theorem 1.4, Note that the functions $s_{i j}(r)=\frac{c_{i j}}{r^{j}}$, where $c_{i j}$ are the operators from interpolation conditions (1.4), satisfy conditions (2.10) in Lemma 2.2 Therefore, there exists $r \in(0,1)$ such that the corresponding Carathéodory-Pick operator $P_{\Sigma(r)}$ is strictly positive definite. Then, by Theorem 2.1, there are infinitely many (rational) Schur functions $h \in \mathcal{S}(\mathcal{X}, \mathcal{Y})$ such that conditions (2.6) are satisfied for $z_{i}=r t_{i}$ and $s_{j}\left(z_{i}\right)=\frac{c_{i j}}{r^{j}}$ :

$$
\frac{h^{(j)}\left(r t_{i}\right)}{j !}=\frac{c_{i j}}{r^{j}} \quad \text { for } i=1, \ldots, k ; j=0, \ldots, n_{i} .
$$

For each such function $h$, define $f(z):=h(r z)$ which is also a (rational) Schur function; in fact $f$ is analytic and $\|f(z)\| \leq 1$ on $\{z:|z|<1 / r\} \supset \mathbb{D}$. Since $f^{(j)}(z)=r^{j} h^{(j)}(r z)$, conditions (2.11) can be written in terms of $f$ as

$$
\frac{f^{(j)}\left(t_{i}\right)}{j !}=c_{i j} \quad \text { for } i=1, \ldots, k ; j=0, \ldots, n_{i},
$$

which means that $f$ satisfies conditions (1.4) and since the above construction guarantees that there are infinitely many such functions, the proof is completed.

\section{Proof of Lemma 2.2}

This last section can be considered as the appendix containing the proof of the auxiliary Lemma 2.2. First we note that the unique solution $P_{\Sigma(r)}$ of the Stein equation (2.9) can be represented in the integral form as

$$
P_{\Sigma(r)}=\int_{\mathbb{T}}\left(\lambda-\mathcal{J}_{\mathbf{n}}(r \mathbf{t})\right)^{-1}\left(E_{\mathbf{n}} E_{\mathbf{n}}^{*}-C_{\mathbf{n}}(r) C_{\mathbf{n}}(r)^{*}\right)\left(\bar{\lambda}-\mathcal{J}_{\mathbf{n}}(r \mathbf{t})^{*}\right)^{-1} m(d \lambda)
$$

where $m(d \lambda)$ is the normalized Lebesgue measure on $\mathbb{T}$ (see [2, Theorem A.2.1]). In (3.1) and in what follows we write $(\lambda-\mathcal{J})^{-1}$ rather than $(\lambda I-\mathcal{J})^{-1}$. The above integral makes sense whenever $\mathbf{t} \in \mathbb{T}^{k}$ and $r \in(0,1)$ and it is not difficult to verify that $P_{\Sigma(r)}$ defined by (3.1) indeed satisfies the Stein equation (2.9). Let us represent $P_{\Sigma(r)}$ in the block form

$$
P_{\Sigma(r)}=\left[\begin{array}{cc}
A(r) & B(r)^{*} \\
B(r) & D(r)
\end{array}\right] \text { where } A(r) \in \mathcal{L}(\mathcal{Y}) .
$$

It is readily seen (for example, upon comparing the top diagonal block entries in (2.9)) that

$$
A(r)=\frac{I_{\mathcal{Y}}-s_{10}(r) s_{10}(r)^{*}}{1-r^{2}} .
$$


Let us assume that $n_{1} \geq 1$ and that the conditions (2.10) are in force. Then in particular, $A(r)$ is (boundedly) invertible for every $r \in(\rho, 1)$ and we can introduce

$$
S(r)=D(r)-B(r) A(r)^{-1} B(r)^{*},
$$

the Schur complement of $A(r)$ in $P_{\Sigma(r)}$. As we will show in Lemma 3.3 below, $S(r)$ is congruent to the Carathéodory-Pick operator $P_{\widetilde{\Sigma}(r)}$ associated with the data

$$
\widetilde{\Sigma}(r)=\left\{\mathbf{z}=r \mathbf{t}, \mathbf{m}, \widetilde{C}_{\mathbf{m}}(r)\right\}
$$

based on the original collection of points $\mathbf{t}=\left(t_{1}, \ldots, t_{k}\right)$, on the tuple

$$
\mathbf{m}=\left\{m_{1}, \ldots, m_{k}\right\}: m_{1}=n_{1}-1 \text { and } m_{i}=n_{i}(i=2, \ldots, k),
$$

and on an operator $\widetilde{C}_{\mathbf{m}}(r)$ with the block entries $\widetilde{s}_{i, j} \in \mathcal{L}(\mathcal{X}, \mathcal{Y})$ defined below (see formula (3.16) ) in terms of the original operators $s_{i, j}$ from (2.7). To construct these operators we will use the entries in the block decompositions

$$
\mathcal{J}_{\mathbf{n}}(r \mathbf{t})=\left[\begin{array}{cc}
r t_{1} I_{\mathcal{Y}} & 0 \\
F & \mathcal{J}
\end{array}\right], \quad C_{\mathbf{n}}(r)=\left[\begin{array}{c}
s(r) \\
C(r)
\end{array}\right], \quad E_{\mathbf{n}}=\left[\begin{array}{c}
I_{\mathcal{Y}} \\
E
\end{array}\right]
$$

conformal with (3.2), where we have set for short

$$
\mathcal{J}:=\mathcal{J}_{\mathbf{m}}(r \mathbf{t}) \quad \text { and } \quad s_{1,0}(r):=s(r) .
$$

The construction will be carried out in two steps and pointwise (i.e., for each fixed $r$ ); at this point dependence on $r$ can be dropped from the notation. Making use of decompositions (3.7) and of notation (3.8) we first define the operator

$$
G=F\left(I-s s^{*}\right)^{\frac{1}{2}}+\left(r t_{1}-\mathcal{J}\right)\left(E-C s^{*}\right)\left(I-s s^{*}\right)^{-\frac{1}{2}}
$$

and decompose it as

$$
G=\left[\begin{array}{c}
G_{1} \\
\vdots \\
G_{k}
\end{array}\right], \quad \text { where } \quad G_{i}=\left[\begin{array}{c}
g_{i, 0} \\
\vdots \\
g_{i, m_{i}}
\end{array}\right]: \mathcal{Y} \rightarrow \mathcal{Y}^{m_{i}+1}
$$

Explicit formulas for the blocks $g_{i, j}$ can be obtained upon substituting explicit formulas for $F, C$ and $E$ into (3.9). For the top components $g_{i, 0}$ in $G_{i}$, we have

$$
\begin{aligned}
g_{1,0} & =\left(I-s s^{*}\right)^{\frac{1}{2}}, \\
g_{i, 0} & =r\left(t_{1}-t_{i}\right)\left[I-s_{i, 0} s^{*}\right]\left(I-s s^{*}\right)^{-\frac{1}{2}} \quad(i=2, \ldots, k) .
\end{aligned}
$$

Next we introduce

$$
\mathbf{G}=\left[\begin{array}{ccc}
\mathbf{G}_{1} & & 0 \\
& \ddots & \\
0 & & \mathbf{G}_{k}
\end{array}\right] \text { where } \mathbf{G}_{i}=\left[\begin{array}{cccc}
g_{i, 0} & 0 & \ldots & 0 \\
g_{i, 1} & g_{i, 0} & \ddots & \vdots \\
\vdots & \ddots & \ddots & 0 \\
g_{i, m_{i}} & \ldots & g_{i, 1} & g_{i, 0}
\end{array}\right]
$$

is the lower triangular Toeplitz block operator so that $\mathbf{G}_{i}$ commutes with $\mathcal{J}_{m_{1}}\left(z_{i}\right)$ and equality $\mathbf{G}_{i} E_{m_{i}}=G_{i}$ holds by construction, for $i=1, \ldots, k$. Therefore, we also have

$$
\mathbf{G} \mathcal{J}=\mathcal{J} \mathbf{G} \quad \text { and } \quad \mathbf{G} E_{\mathbf{m}}=G
$$

where $G$ is given in (3.10) and $E_{\mathbf{m}}$ is associated to $\mathbf{m}$ of the form (3.6) via the second formula in (2.4). Recall that $\mathbf{G}$ constructed above is a function of $r$. The next remark establishes some uniform estimates needed for the subsequent analysis. 
Remark 3.1. Conditions (2.10) guarantee that $\|\mathbf{G}(r)\|$ and $\left\|\mathbf{G}(r)^{-1}\right\|$ are bounded uniformly with respect to $r \in(\rho, 1)$.

Proof. Due to the structure (3.13) of $\mathbf{G}$ and in view of (3.10), it suffices to show that $\|G(r)\|$ and $\left\|g_{i, 0}(r)^{-1}\right\|$ (for $i=1, \ldots, k$ ) are uniformly bounded on $(\rho, 1)$. To this end, we first note that by the first condition in (2.10),

$$
\left\|\left(I-s(r) s(r)^{*}\right)^{-\frac{1}{2}}\right\| \leq \frac{1}{\sqrt{1-\delta^{2}}}, \quad\left\|\left(I-s_{i, 0}(r) s(r)^{*}\right)^{-1}\right\| \leq \frac{1}{1-\delta^{2}} .
$$

Since $\|C(r)\| \leq M$ by the second condition in (2.10), and since $\|F\|=\|E\|=1$ and $\left\|\mathcal{J}_{\mathbf{m}}(r \mathbf{t})\right\| \leq 2$ by definitions, the uniform bound

$$
\|G(r)\| \leq 1+\frac{3(1+\delta M)}{\sqrt{1-\delta^{2}}}
$$

follows from (3.9) by the triangle inequality. Furthermore, comparing (3.11) and the first relation in (3.15) we get $\left\|g_{1,0}(r)^{-1}\right\| \leq\left(1-\delta^{2}\right)^{-\frac{1}{2}}$, while (3.12) along with the second relation in (3.15) gives

$$
\left\|g_{i, 0}(r)^{-1}\right\| \leq \frac{1}{\rho\left|t_{1}-t_{i}\right|} \cdot \frac{1}{1-\delta^{2}}
$$

and completes the proof.

Now we complete the construction of the data $\widetilde{\Sigma}(r)$ by defining the operator

$$
\widetilde{C}_{\mathbf{m}}(r)=\left(I-r \bar{t}_{1} \mathcal{J}\right) \mathbf{G}(r)^{-1}\left[E s_{1,0}(r)-C(r)\right]\left(I-s_{1,0}(r)^{*} s_{1,0}(r)\right)^{-\frac{1}{2}}
$$

and decomposing it conformally with (3.10) (again dropping the variable $r$ for short) as

$$
\widetilde{C}_{\mathbf{m}}=\left[\begin{array}{c}
\widetilde{C}_{m_{1}} \\
\vdots \\
\widetilde{C}_{m_{k}}
\end{array}\right], \quad \text { where } \quad \widetilde{C}_{m_{i}}=\left[\begin{array}{c}
\widetilde{s}_{i, 0} \\
\vdots \\
\widetilde{s}_{i, m_{i}}
\end{array}\right]
$$

In some more detail (again we write $s$ for $s_{1,0}$ ):

$$
\widetilde{C}_{m_{1}}=\left(I-r \bar{t}_{1} \mathcal{J}_{m_{1}}\left(r t_{1}\right)\right) \mathbf{G}_{1}^{-1}\left[\begin{array}{c}
s_{1,1} \\
s_{1,2} \\
\vdots \\
s_{1, n_{1}}
\end{array}\right] s^{*}\left(I-s s^{*}\right)^{-\frac{1}{2}}
$$

and

$$
\widetilde{C}_{m_{i}}=\left(I-r \bar{t}_{1} \mathcal{J}_{n_{i}}\left(r t_{i}\right)\right) \mathbf{G}_{i}^{-1}\left[E_{n_{i}} s-C_{n_{i}}\right]\left(I-s^{*} s\right)^{-\frac{1}{2}}
$$

for $i=2, \ldots, k$. The top components $\widetilde{s}_{i, 0}$ in the two latter formulas will be of special interest. Due to the lower triangular structure of $\mathbf{G}_{i}$ and $\mathcal{J}_{m_{i}}\left(r t_{i}\right)$, we have by (3.18) and (3.11),

$$
\begin{aligned}
\widetilde{s}_{1,0} & =\left(1-r^{2}\right) g_{1,0}^{-1} s_{1,1} s^{*}\left(I-s s^{*}\right)^{-\frac{1}{2}} \\
& =\left(1-r^{2}\right)\left(I-s s^{*}\right)^{-\frac{1}{2}} s_{1,1} s^{*}\left(I-s s^{*}\right)^{-\frac{1}{2}},
\end{aligned}
$$

and similarly, (3.19) and (3.12) bring us to

$$
\widetilde{s}_{i, 0}=\left(1-r^{2} \bar{t}_{1} t_{i}\right) g_{i, 0}^{-1}\left(s-s_{i, 0}\right)\left(I-s^{*} s\right)^{-\frac{1}{2}}=\frac{1-r^{2} \bar{t}_{1} t_{i}}{r\left(t_{1}-t_{i}\right)} L_{i}
$$


where

$$
L_{i}:=\left(I-s s^{*}\right)^{\frac{1}{2}}\left(I-s_{i, 0} s^{*}\right)^{-1}\left(s-s_{i, 0}\right)\left(I-s^{*} s\right)^{-\frac{1}{2}} \quad(i=2, \ldots, k) .
$$

Remark 3.2. Let us assume that conditions (2.10) are in force for the data $\Sigma(r)$ defined in (2.8). Then the data $\widetilde{\Sigma}(r)$ constructed in (3.5), (3.6), (3.16), satisfies similar conditions: there exist positive numbers $\widetilde{\delta}<1, \widetilde{\rho}<1$ and $\widetilde{M}$ such that

$$
\left\|\widetilde{s}_{i, 0}(r)\right\| \leq \widetilde{\delta} \text { and }\left\|\widetilde{s}_{i, j}(r)\right\| \leq \widetilde{M}
$$

for all $j=1, \ldots, m_{i}, i=1, \ldots, k$ and $r \in(\widetilde{\rho}, 1)$.

Proof. Upon making use of Remark 3.1 and of the uniform bounds from its proof we conclude from (3.16) that

$$
\left\|\widetilde{C}_{\mathbf{m}}(r)\right\| \leq 3 \cdot\left\|\mathbf{G}(r)^{-1}\right\| \cdot \frac{\delta+M}{\sqrt{1-\delta^{2}}}=: \widetilde{M}<\infty
$$

and thus we have at least the same uniform bound for the block entries $\widetilde{s}_{i, j}$ in $\widetilde{C}_{\mathbf{m}}(r)$ which proves the second series of bounds in (3.23). To check the first series of bounds in (3.23), we start with identity

$$
I-L_{i} L_{i}^{*}=\left(I-s s^{*}\right)^{\frac{1}{2}}\left(I-s_{i, 0} s^{*}\right)^{-1}\left(I-s_{i, 0} s_{i, 0}^{*}\right)\left(I-s s_{i, 0}^{*}\right)^{-1}\left(I-s s^{*}\right)^{\frac{1}{2}}
$$

(where $L_{i}$ is defined in (3.22) ) verification of which is straightforward. Due to the first bound in (2.10),

$$
I-s_{i, 0} s_{i, 0}^{*} \geq\left(1-\delta^{2}\right) \cdot I \quad(i=1, \ldots, k)
$$

and by the triangle inequality, $1-\delta^{2} \leq\left\|I-s s_{i, 0}^{*}\right\|<2$, so that

$$
\left(I-s_{i, 0} s_{1,0}^{*}\right)^{-1}\left(I-s_{1,0} s_{i, 0}^{*}\right)^{-1} \geq \frac{1}{4} \cdot I .
$$

Therefore,

$$
\begin{aligned}
I-L_{i} L_{i}^{*} & \geq\left(1-\delta^{2}\right)\left(I-s s^{*}\right)^{\frac{1}{2}}\left(I-s_{i, 0} s^{*}\right)^{-1}\left(I-s s_{i, 0}^{*}\right)^{-1}\left(I-s s^{*}\right)^{\frac{1}{2}} \\
& \geq \frac{1-\delta^{2}}{4} \cdot\left(I-s s^{*}\right) \geq \frac{\left(1-\delta^{2}\right)^{2}}{4} \cdot I
\end{aligned}
$$

and thus,

$$
\left\|L_{i}(r)\right\| \leq\left(1-\frac{\left(1-\delta^{2}\right)^{2}}{4}\right)^{\frac{1}{2}}=: \delta_{1} .
$$

On the other hand, the identity

$$
\Psi(r):=\left|\frac{r\left(t_{1}-t_{i}\right)}{1-r^{2} \bar{t}_{1} t_{i}}\right|^{2}=1-\frac{\left(1-r^{2}\right)^{2}}{\left|1-r^{2} \bar{t}_{1} t_{i}\right|^{2}}
$$

shows that the continuous function $\Psi(r)$ is less than one on $(\rho, 1)$ and tends to one as $r \rightarrow 1$. Pick $\delta_{2} \in\left(\delta_{1}, 1\right)$ and choose $\rho_{1}$ such that $\Psi(r)>\delta_{2}$ whenever $r \in\left(\rho_{1}, 1\right)$. Now we get from (3.21) and (3.24) that for every $r \in\left(\rho_{1}, 1\right)$,

$$
\left\|\widetilde{s}_{i, 0}(r)\right\|=\frac{\left\|L_{i}\right\|}{\Psi(r)}<\frac{\delta_{1}}{\delta_{2}}=: \widetilde{\delta}<1 \quad \text { for } i=2, \ldots, k .
$$

Finally, it follows from (3.20) by (3.15) and uniform bounds $\|s(r)\| \leq \delta$ and $\left\|s_{1,1}(r)\right\| \leq M$, that

$$
\left\|\widetilde{s}_{1,0}(r)\right\| \leq\left(1-r^{2}\right) \cdot \frac{\delta M}{1-\delta^{2}} .
$$


Choosing $\rho_{2}$ such that $\left(1-r^{2}\right) \frac{\delta M}{1-\delta^{2}}<\widetilde{\delta}$ for every $r \in\left(\rho_{2}, 1\right)$ and setting $\widetilde{\rho}=$ $\max \left\{\rho_{1}, \rho_{2}\right\}$, we get the first series of uniform bounds in (3.21).

Lemma 3.3. Let us assume that conditions (2.10) are in force for the data $\Sigma(r)$ defined in (2.8) and let $S(r)$ be the Schur complement of the block $A(r)$ in the decomposition (3.2) of the associated Carathéodory-Pick operator $P_{\Sigma(r)}$. Then for every fixed $r \in(\rho, 1)$, the operator

$$
\widetilde{S}(r):=\mathbf{G}(r)^{-1}\left(I-r \bar{t}_{1} \mathcal{J}_{\mathbf{m}}(r \mathbf{t})\right) S(r)\left(I-r t_{1} \mathcal{J}_{\mathbf{m}}(r \mathbf{t})^{*}\right)\left(\mathbf{G}(r)^{-1}\right)^{*}
$$

is equal to the Carathéodory-Pick operator $P_{\widetilde{\Sigma}(r)}$ associated with the data (3.5) where $\mathbf{m}, \widetilde{C}_{\mathbf{m}}$ and $\mathbf{G}$ are defined in (3.6), (3.16) and (3.13), respectively.

Proof. It suffices to show that $\widetilde{S}(r)$ admits the integral representation

$$
\widetilde{S}(r)=\int_{\mathbb{T}}\left(\lambda-\mathcal{J}_{\mathbf{m}}(r \mathbf{t})\right)^{-1}\left(E_{\mathbf{m}} E_{\mathbf{m}}^{*}-\widetilde{C}_{\mathbf{m}}(r) \widetilde{C}_{\mathbf{m}}(r)^{*}\right)\left(\bar{\lambda}-\mathcal{J}_{\mathbf{m}}(r \mathbf{t})^{*}\right)^{-1} m(d \lambda)
$$

since the right hand side integral represents $P_{\widetilde{\Sigma}(r)}$, by a virtue of (3.1). Since $r$ is fixed, dependence of some items on $r$ will be dropped for short. First we multiply both parts in the integral representation (3.1) by the operator

$$
T=\left[\begin{array}{ll}
-B A^{-1} & I
\end{array}\right]
$$

on the left and by $T^{*}$ on the right. Since $T P_{\Sigma} T^{*}=S$, we have

$$
S=\int_{\mathbb{T}} T\left(\lambda-\mathcal{J}_{\mathbf{n}}(r \mathbf{t})\right)^{-1}\left(E_{\mathbf{n}} E_{\mathbf{n}}^{*}-C_{\mathbf{n}}(r) C_{\mathbf{n}}(r)^{*}\right)\left(\bar{\lambda}-\mathcal{J}_{\mathbf{n}}(r \mathbf{t})^{*}\right)^{-1} T^{*} m(d \lambda)
$$

To simplify the integrand, we will use block decompositions (3.7) conformal with (3.2) and notation (3.8). Note that by (3.7) and (3.3),

$$
E_{\mathbf{n}} E_{\mathbf{n}}^{*}-C_{\mathbf{n}}(r) C_{\mathbf{n}}(r)^{*}=\left[\begin{array}{cc}
\left(1-r^{2}\right) A & X^{*} \\
X & E E^{*}-C C^{*}
\end{array}\right]
$$

where

$$
X:=E-C s^{*} .
$$

Substituting decompositions (3.2) and (3.7) into (2.3) and comparing the off diagonal blocks we get

$$
B-r \bar{t}_{1} F A-r \bar{t}_{1} \mathcal{J} B=E-C s^{*}=X
$$

which implies

$$
B=\left(I-r \bar{t}_{1} \mathcal{J}\right)^{-1}\left[\bar{z}_{1} F A+X\right] .
$$

Therefore,

$$
\begin{aligned}
F-(\lambda-\mathcal{J}) B A^{-1} & =\left(I-r \bar{t}_{1} \mathcal{J}\right)^{-1}\left(\left(I-r \bar{t}_{1} \mathcal{J}\right) F-(\lambda-\mathcal{J})\left[r \bar{t}_{1} F+X A^{-1}\right]\right) \\
(3.32) & =\left(I-r \bar{t}_{1} \mathcal{J}\right)^{-1}\left(\left(1-r \lambda \bar{t}_{1}\right) F-(\lambda-\mathcal{J}) X A^{-1}\right) .
\end{aligned}
$$

The first decomposition in (3.7) gives

$$
\left(\lambda-\mathcal{J}_{\mathbf{n}}(r \mathbf{t})\right)^{-1}=\frac{1}{\lambda-r t_{1}}\left[\begin{array}{cc}
I_{\mathcal{Y}} & 0 \\
(\lambda-\mathcal{J})^{-1} F & (\lambda-\mathcal{J})^{-1}
\end{array}\right]
$$


which together with (3.5), (3.27) and (3.31) leads us to

$$
\begin{aligned}
& T\left(\lambda-\mathcal{J}_{\mathbf{n}}(r \mathbf{t})\right)^{-1}=\frac{1}{\lambda-r t_{1}}\left[\begin{array}{ll}
-B A^{-1} & I
\end{array}\right]\left[\begin{array}{cc}
I_{\mathcal{Y}} & 0 \\
(\lambda-\mathcal{J})^{-1} F & (\lambda-\mathcal{J})^{-1}
\end{array}\right] \\
& =\frac{1}{\lambda-r t_{1}}(\lambda-\mathcal{J})^{-1}\left[\begin{array}{ll}
F-(\lambda-\mathcal{J}) B A^{-1} & I
\end{array}\right] \\
& =\left(I-r \bar{t}_{1} \mathcal{J}\right)^{-1}(\lambda-\mathcal{J})^{-1} \\
& \times\left[\frac{1-r \lambda \bar{t}_{1}}{\lambda-r t_{1}} F-\frac{1}{\lambda-r t_{1}}(\lambda-\mathcal{J}) X A^{-1} \quad I-r \bar{t}_{1} \mathcal{J}\right] .
\end{aligned}
$$

Now we substitute the latter equality and (3.29) into (3.28) and multiply the obtained equality by the operator $\mathbf{G}^{-1}\left(I-r \bar{t}_{1} \mathcal{J}\right)$ on the left and by its adjoint on the right. On account of (3.14) and (3.25), we have

$$
\widetilde{S}=\int_{\mathbb{T}}(\lambda-\mathcal{J})^{-1} \mathbf{G}^{-1} K(\lambda)\left(\mathbf{G}^{*}\right)^{-1}\left(\bar{\lambda}-\mathcal{J}^{*}\right)^{-1} m(d \lambda)
$$

where

$$
\begin{aligned}
K(\lambda)= & {\left[\begin{array}{cc}
\frac{1-r \lambda \bar{t}_{1}}{\lambda-r t_{1}} F-\frac{1}{\lambda-r t_{1}}(\lambda-\mathcal{J}) X A^{-1} & I-r \bar{t}_{1} \mathcal{J}
\end{array}\right] } \\
& \times\left[\begin{array}{cc}
\left(1-r^{2}\right) A & X^{*} \\
X & E E^{*}-C C^{*}
\end{array}\right]\left[\begin{array}{c}
\frac{1-r \bar{\lambda} t_{1}}{\lambda-r \bar{t}_{1}} F^{*}-\frac{1}{\lambda-r \bar{t}_{1}} A^{-1} X^{*}\left(\bar{\lambda}-\mathcal{J}^{*}\right) \\
I-r t_{1} \mathcal{J}^{*}
\end{array}\right] .
\end{aligned}
$$

Expanding the right hand side expression and subsequent simplifying (with taking advantage of the fact that $|\lambda|=1$ ) brings us to

$$
K(\lambda)=G G^{*}-\left(I-r \bar{t}_{1} \mathcal{J}\right)(E s-C)\left(s^{*} E^{*}-C^{*}\right)\left(I-r t_{1} \mathcal{J}^{*}\right)
$$

and shows that $K(\lambda)$ does not depend on $\lambda$. It remains to multiply the latter equality by $\mathbf{G}^{-1}$ on the left, by $\left(\mathbf{G}^{*}\right)^{-1}$ on the right and to use formulas (3.16) and (3.14) to get

$$
\mathbf{G}^{-1} K\left(\mathbf{G}^{*}\right)^{-1}=E_{\mathbf{m}} E_{\mathbf{m}}^{*}-\widetilde{C}_{\mathbf{m}}(r) \widetilde{C}_{\mathbf{m}}(r)^{*}
$$

Substituting the latter equality into (3.33) we get (3.26) and complete the proof.

Proof of Lemma 2.2, The proof will be obtained by induction in $N=|\mathbf{n}|=n_{1}+$ $\ldots+n_{k}$. If $N=0$, then $P_{\Sigma(r)}$ reduces to the Pick matrix whose diagonal block entries satisfy

$$
P_{i i}(r)=\frac{I-s_{i 0}(r) s_{i 0}(r)}{1-r^{2}} \geq \frac{\delta}{1-r^{2}} \cdot I
$$

whereas the off diagonal blocks are subject to

$$
\left\|P_{i j}(r)\right\|=\left\|\frac{I-s_{i 0}(r) s_{j 0}(r)}{1-r^{2} t_{i} \bar{t}_{j}}\right\| \leq \frac{2}{\left|\sin \left(\arg \left(t_{i}-t_{j}\right)\right)\right|} .
$$

Now it is clear that $P_{\Sigma(r)}$ is strictly positive if $r$ is sufficiently close to one. Let us assume that the statement of the lemma holds for every choice of the data $\Sigma(r)$ of the form (2.8) with $|\mathbf{n}| \leq N$ and satisfying conditions (2.10) for some positive $M$ and $\delta$. Pick data $\Sigma(r)$ of the form (2.8) satisfying (2.10) and with $|\mathbf{n}|=N+1$. To complete the proof by induction argument, we have to show that $P_{\Sigma(r)}$ is s.p.d. for every $r<1$ sufficiently close to one. To this end, note that one of the components $n_{1}, \ldots, n_{k}$ in $\mathbf{n}$ must be positive; without loss of generality we assume that $n_{1} \geq 1$. Using decomposition (3.2) of $P_{\Sigma(r)}$ in which the block $A$ is s.p.d. by (2.10), we construct the date $\left.\widetilde{\Sigma}(r)=\left\{\mathbf{z}=r \mathbf{t}, \mathbf{m}, \widetilde{C}_{\mathbf{m}}(r)\right\}\right\}$ with $\mathbf{m}$ and $\widetilde{C}_{\mathbf{m}}(r)$ defined as in (3.6) and (3.16), (3.17). By Remark 3.2, the block entries $\widetilde{s}_{i j}(r)$ of $\widetilde{C}_{\mathbf{m}}(r \mathbf{t})$ 
meet conditions (3.23) and since $|\mathbf{m}|=N$, we can use the induction hypothesis to conclude that the associated Carathéodory-Pick operator $P_{\widetilde{\Sigma}(r)}$ is s.p.d. for every $r \in(\widetilde{\rho}, 1)$. Now we again use (3.2) to write the factorization formula

$$
P_{\Sigma(r)}=\left[\begin{array}{cc}
I & 0 \\
B(r) A(r)^{-1} & I
\end{array}\right]\left[\begin{array}{cc}
A(r) & 0 \\
0 & S(r)
\end{array}\right]\left[\begin{array}{cc}
I & A(r)^{-1} B(r)^{*} \\
0 & I
\end{array}\right],
$$

where $S(r)$ is defined in (3.4), which implies (since $A$ is s.p.d.) that $P_{\Sigma}(r)$ is s.p.d. if and only if $S(r)$ is s.p.d. By Lemma 3.3 .

$$
\mathbf{G}(r)^{-1}\left(I-r \bar{t}_{1} \mathcal{J}_{\mathbf{m}}(r \mathbf{t})\right) S\left(I-r t_{1} \mathcal{J}_{\mathbf{m}}(r \mathbf{t})^{*}\right)\left(\mathbf{G}(r)^{-1}\right)^{*}=P_{\widetilde{\Sigma}(r)}
$$

and since the operator $\mathbf{G}(r)^{-1}\left(I-r \bar{t}_{1} \mathcal{J}_{\mathbf{m}}(r \mathbf{t})\right)$ is boundedly invertible for every fixed $r \in(\widetilde{\rho}, 1), S(r)$ (and therefore, $P_{\Sigma}(r)$ as well) is s.p.d. whenever $P_{\widetilde{\Sigma}(r)}$ is; that is, in particular, for every $r \in(\widetilde{\rho}, 1)$.

\section{REFERENCES}

1. J. A. Ball, Interpolation problems of Pick-Nevanlinna and Loewner type for meromorphic matrix functions, Integral Equations Operator Theory 6 (1983), 804-840. MR719106|(85k:30054)

2. J. A. Ball, I. Gohberg and L. Rodman, Interpolation of Rational Matrix Functions, OT45, Birkhäuser-Verlag, Basel-Boston, 1990. MR.1083145 (92m:47027)

3. J. A. Ball and J. W. Helton, Interpolation problems of Pick-Nevanlinna and Loewner types for meromorphic matrix functions: parametrization of the set of all solutions, Integral Equations Operator Theory 9 (1986), 155-203. MR837514 (87j:30085)

4. V. Bolotnikov and H. Dym, On boundary interpolation for matrix valued Schur functions, Mem. Amer. Math. Soc. 181 (2006), no. 856. MR2214130

5. V. Bolotnikov and A. Kheifets, The Carathéodory-Julia theorem and related boundary interpolation problems, Recent Advances in Matrix and Operator Theory (J. A. Ball, Y. Eidelman, J. W. Helton, V. Olshevsky, and J. Rovnyak, eds.), Operator Theory: Advances and Applications 179, pp. 63-102, Birkhäuser-Verlag, Basel, 2007.

6. C. Carathéodory, Über die Winkelderivierten von beschränkten Funktionen, Sitzungber. Preuss. Akad. Wiss. (1929), 39-52.

7. G. Julia, Extension d'un lemma de Schwarz, Acta Math. 42 (1920), 349-355.

8. C. Foias, A. E. Frazho, I. Gohberg and M. A. Kaashoek, Metric constrained interpolation, commutant lifting and systems, Operator Theory: Advances and Applications 100, Birkhäuser-Verlag, Basel, 1998. MR.1635831 (99i:47027)

9. D. R. Georgijević, Solvability condition for a boundary value interpolation problem of Loewner type, J. Anal. Math. 74 (1998), 213-234. MR1631662 (99g:47026)

10. I. Gohberg, M. A. Kaashoek and A. L. Sakhnovich, Taylor coefficients of a pseudo-exponential potential and the reflection coefficient of the corresponding canonical system, Math. Nachr. 278 (2005), no. 12-13, 1579-1590. MR2169701 (2006i:34169)

11. P. P. Khargonekar and A. Tannenbaum, Non-Euclidean metrics and the robust stabilization of systems with parameter uncertainty, IEEE Trans. Automat. Control 30 (1985), no. 10, 1005-1013. MR804138 (86m:93066)

12. I. V. Kovalishina, A multiple boundary interpolation problem for contracting matrix functions in the unit disk, J. Soviet Math. 52(6) (1990), 3467-3481. MR.1009144 (90i:41007)

13. D. Sarason, Nevanlinna-Pick interpolation with boundary data, Integral Equations Operator Theory 30 (1998), 231-250. MR1607902 (98m:47017)

14. I. Schur, Über Potenzreihen, die im Innern des Einheitskreises beschränkt sind, J. Reine Angew. Math. 147 (1917), 205-232.

Department of Mathematics, College of William and Mary, Williamsburg, Virginia $23187-8795$ 